\title{
Clinical features of malignant melanoma of the finger and therapeutic efficacies of different treatments
}

\author{
ZUOZHANG YANG, LIN XIE, YUNCHAO HUANG, HONGPU SUN, \\ TAO YUAN, XIANG MA, CONGGUO JING and PENGJIE LIU \\ Department of Orthopedics, Tumor Hospital of Yunnan Province, The Third Affiliated Hospital \\ of Kunming Medical College, Kunming, Yunnan 650118, P.R. China
}

Received May 31, 2011; Accepted June 1, 2011

DOI: $10.3892 / 01.2011 .324$

\begin{abstract}
The purpose of this study was to explore the clinical features of malignant melanoma of the finger and therapeutic efficacies of different treatments. The clinical data of 22 patients with malignant melanoma of the finger (confirmed by pathological examination), admitted and treated in our hospital between February 1995 and October 2007, were analyzed retrospectively. The primary site of the tumor was the thumb in 12 cases, index finger in 3 cases, middle finger in 3 cases, ring finger in 2 cases and little finger in 2 cases. The most common presenting symptoms were pain and black patches on the fingers. A total of 15 of the 22 patients had subungual disease, 12 had a history of trauma and 2 had osteolytic bone lesions of the phalanx. The main treatments employed were surgery, chemotherapy and immunotherapy. Finger amputation was performed for all 22 patients and axillary lymph node dissection in the same side was performed for 13 patients. Nineteen cases were followed up for 1-10 years with an average of 5.5 years. Three patients were lost to followup 2 years after treatment. The 1-, 3- and 5-year survival rates were $86.4 \%(19 / 22), 42.1 \%(12 / 19)$ and $31.2 \%$ (6/19), respectively. In conclusion, malignant melanoma of the finger is a rarely occurring tumor. Comprehensive treatment, including surgery, chemotherapy and immunotherapy, is the key approach for malignant melanoma of the finger. Prognosis of the disease is associated with the size of the tumor, depth of infiltration and clinical stages.
\end{abstract}

\section{Introduction}

Malignant melanoma is a class of malignant tumors derived from neural crest cells. Its rate of occurrence is low, accounting

Correspondence to: Dr Zuozhang Yang, Department of Orthopedics, Tumor Hospital of Yunnan Province, The Third Affiliated Hospital of Kunming Medical College, Kunming, Yunnan 650118, P.R. China E-mail: yangzuozhang@163.com

Key words: finger, malignant melanoma, tumor, clinical analysis, clinical pathology for 1-3\% of total malignant tumors (1). The occurrence of malignant melanoma in fingers is particularly rare. Melanoma of the finger is a highly malignant tumor that is restricted to specific areas of the finger. In the past, the incidence of malignant melanoma was low, but recently its incidence has been increase due to lifestyle changes and advances in diagnosis. Chemotherapy has a low efficacy and poor prognosis for malignant melanoma. It is effective for some patients, with an efficiency rate of $20-30 \%$, but the effective period is short (2). Subsequently, it was found that malignant melanoma had high immunogenicity. Therefore, biological therapy for malignant melanoma has been widely applied. For example, the efficiency rate of interferon- $\alpha$ (INF $\alpha$ ) and interleukin-2 (IL-2) in the treatment of malignant melanoma ranged from 10 to $20 \%$, and the life span has been extended for those patients (3). Investigators have found that the combination treatment of chemotherapy and cytokines (i.e., biochemotherapy) treatment improves efficacy, leading to an efficiency rate of $60-70 \%$. Biochemical treatment refers to the appropriate utilization of biological and chemical substances to achieve an additive effect, enhancement of the healing rate and improvement in quality of life (4). Early diagnosis and comprehensive treatment are key factors involved in improving survival rate. In this study, 22 cases of malignant melanoma of the finger were treated between February 1995 and October 2007. The clinical features and treatment approaches for these patients are retrospectively reported.

\section{Patients and methods}

Patients. A total of 22 patients were included in this study. Of these patients, 16 were male and 6 female. The patient age ranged from 24 to 76 years with a median age of 48.3. The thumb was the primary tumor site in 12 cases, the index finger in 3 cases, the middle finger in 3 cases, the ring finger in 2 cases and the little finger in 2 cases. The occurrence of black patches and pain in the finger were the common initial symptoms (Fig. 1). Of the 22 patients, 13 had prolonged skin ulcers in restricted areas of the finger (Fig. 2), 15 patients had subungual lesions (Fig. 3), 12 patients had a history of trauma and 11 patients had a history of nail pull-out (Fig. 4). Osteolytic changes in the phalanx were found in 2 cases by X-ray examination. Malignant melanoma was confirmed in all patients by pathological examination. The longest diameter of the primary tumor ranged from 


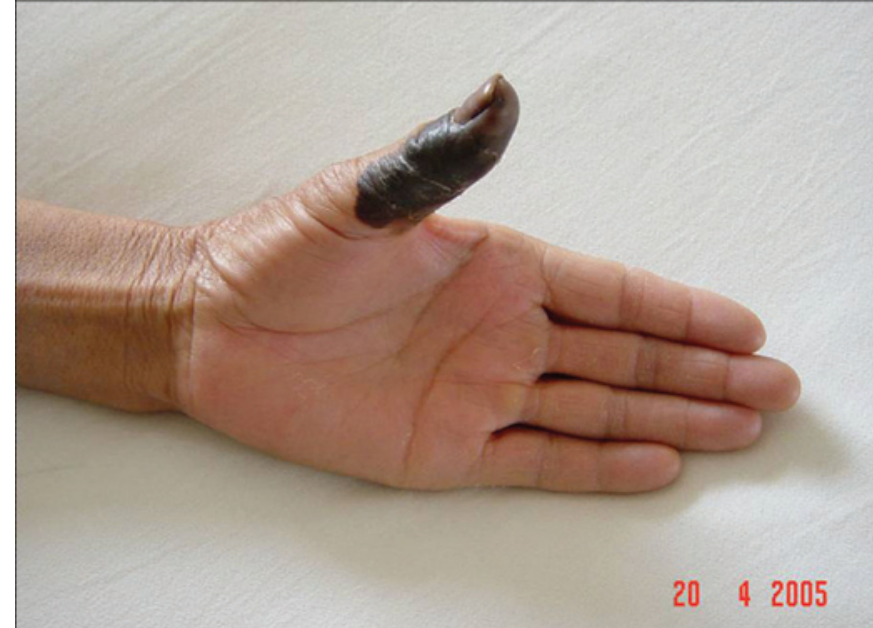

Figure 1. Malignant melanoma in the left thumb exhibiting dark specks of skin.

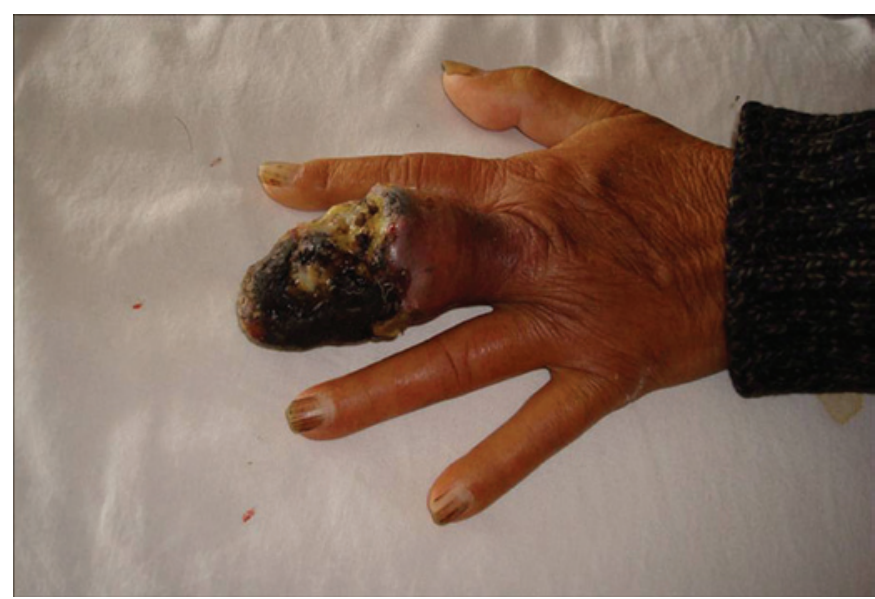

Figure 2. Malignant melanoma in the left middle finger accompanied by ulcer.

0.6 to $4.2 \mathrm{~cm}$ with a median value of $1.9 \mathrm{~cm}$. B-ultrasonic examinations showed that 14 patients had lymphadenectasis in the same side of the diseased finger. Based on the AJCC2000 melanoma staging standard (5), the patients in this study were divided into stage I $(n=3)$, stage II $(n=5)$, stage III $(n=12)$ and stage IV $(n=2)$.

Histological grading. Histological grading was performed according to the Clark grading system (6). Grade I was found in $4.5 \%(1 / 22)$ of the patients. Primary malignant melanoma (grade II, with a tumor thickness of $<0.75 \mathrm{~mm}$ and infiltration in the papillary dermis) was found in $27.3 \%(4 / 22)$ of the patients. Grade III (with a tumor thickness of $>0.65$, but $<1.5 \mathrm{~mm}$ and infiltration of the papillary/reticular layer of the skin) was found in $22.7 \%(5 / 22)$ of the patients. Grade IV (with a tumor thickness of $>1.5$, but $<4.0 \mathrm{~mm}$ and infiltration of the reticular layer of the skin) was found in $31.8 \%(7 / 22)$ of the cases. Grade V (with a tumor thickness of $>4.0 \mathrm{~mm}$ and infiltration of the subcutaneous fat layer or occurrence of satellite lesions within $2 \mathrm{~cm}$ of the primary tumor) was found in $13.6 \%$ $(3 / 22)$ of the patients.

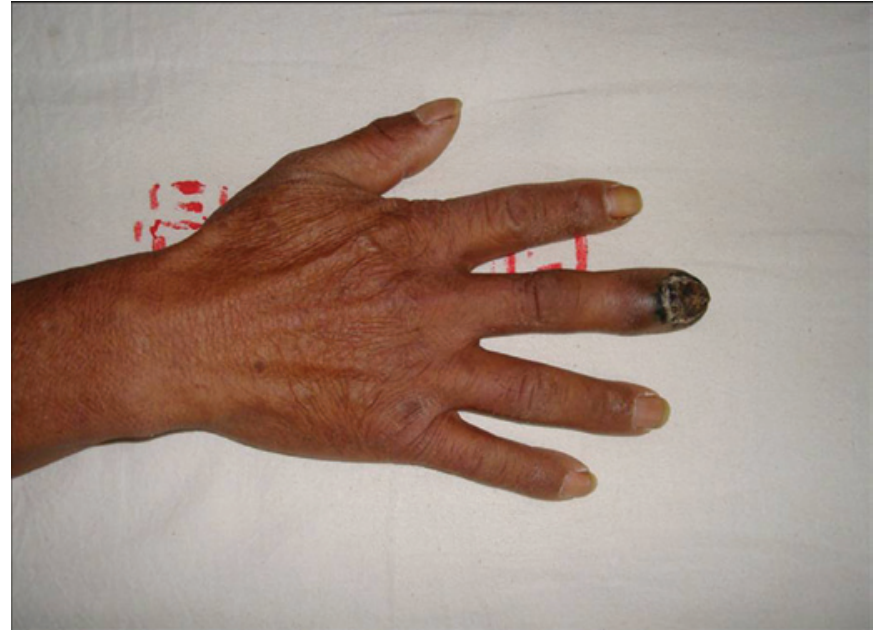

Figure 3. Malignant melanoma in the right middle finger exhibiting subungual lesions.

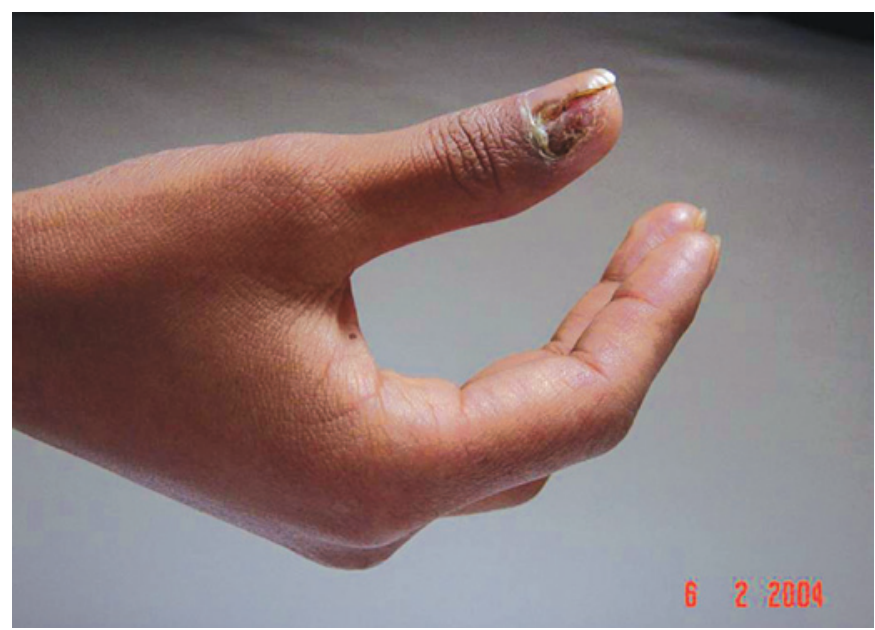

Figure 4. Malignant melanoma in the left thumb with a history of nail pull-out.

Surgical operation. Amputation was performed for 8 patients with tumor stages I and II. The principle of the surgery was expanded removal of the tumor. In order for the finger to have a good appearance following amputation, finger skin was preserved as much as possible prior to stitching, which facilitated the recovery of the finger stump. To achieve good resistance, the palmar skin was used as oval valve to flop over and cover the finger stump from the dorsal side. Amputation as well as axillary lymph node dissection were performed on 12 patients with stage III tumors. For those patients with lymphadenectasis accompanied by skin infiltration, local lymph nodes as well as the subcutaneous tissues were removed in a spindle manner. The axillary lymph nodes in the 12 patients were significantly swollen. The swollen lymph nodes and the surrounding subcutaneous tissues were completely removed. Of these 12 patients, 2 had small satellite lesions surrounding the swollen lymph nodes. For these 2 patients, the surrounding skin and subcutaneous tissue $(>3 \mathrm{~cm})$ were removed. The intermediate dermatome was used to cover the wound surfaces. A negative pressure tube was placed under the skin flap for drainage for 7-10 days. Pathological examination confirmed 
Table I. Relationship of the survival period of patients with finger malignant melanoma and clinical factors.

\begin{tabular}{|c|c|c|c|c|c|c|c|c|}
\hline \multirow[t]{2}{*}{ Factors } & \multirow[t]{2}{*}{ No. } & \multicolumn{5}{|c|}{ Survival time (month) } & \multirow[t]{2}{*}{$\chi^{2}$ value } & \multirow[t]{2}{*}{ P-value } \\
\hline & & Median & SE & $25 \%$ percentiles & $75 \%$ percentiles & QR & & \\
\hline Gender & & & & & & & 0.37 & 0.620 \\
\hline Male & 16 & 30 & 4.02 & 20 & 7 & 13 & & \\
\hline Female & 6 & 24 & 6.32 & 74 & 4 & 70 & & \\
\hline Tumor size (cm) & & & & & & & 8.15 & 0.009 \\
\hline$<2$ & 13 & 32 & 2.61 & 55 & 12 & 43 & & \\
\hline$\geq 2$ & 9 & 15 & 2.48 & 9 & 3 & 6 & & \\
\hline Depth of the infiltration (mm) & & & & & & & 18.10 & 0.000 \\
\hline$<1.5$ & 7 & 46 & 17.68 & 74 & 20 & 54 & & \\
\hline $1.5-4.0$ & 11 & 30 & 9.98 & 16 & 9 & 7 & & \\
\hline$>4.0$ & 4 & 8 & 3.69 & 8 & 3 & 4 & & \\
\hline Metastasis of axillary lymph node & & & & & & & 23.52 & 0.003 \\
\hline Yes & 13 & 21 & 1.82 & 11 & 4 & 3 & & \\
\hline No & 9 & 40 & 0.81 & 62 & 10 & 46 & & \\
\hline Clinical staging (grade) & & & & & & & 24.90 & 0.000 \\
\hline I & 3 & 48 & 18.96 & 8 & 6 & 5 & & \\
\hline II & 5 & 39 & 17.68 & 12 & 7 & 7 & & \\
\hline III & 12 & 18 & 4.85 & 55 & 15 & 40 & & \\
\hline IV & 2 & 7 & 1.36 & 6 & 3 & 3 & & \\
\hline
\end{tabular}

that 11 patients had lymph node metastasis (the number of lymph nodes with tumor metastasis was 2-8) after axillary lymph node removal. Two patients had lymph node reactive hyperplasia. One stage IV patient had malignant melanoma in the left hand, as well as metastasis of the lymph node, liver and lung in the same side. Amputation, axillary lymph node removal and liver intervention therapy were performed for this patient. The remaining stage IV patient only received amputation and systemic chemotherapy.

Chemotherapy. One cycle of pre-operative chemotherapy and 4-6 cycles of postoperative chemotherapy were administered. The BDVP scheme was used for chemotherapy. Briefly, lomustine (CCNU) was used at $150 \mathrm{mg} / \mathrm{m}^{2}$ on day 1 , dacarbazine was used at $200 \mathrm{mg} / \mathrm{m}^{2}$ on days $1-5$, vincristine (VCR) was used at $4 \mathrm{mg} / \mathrm{m}^{2}$ on day 1 and cisplatin (DDP) was used at $30 \mathrm{mg} / \mathrm{m}^{2}$ on days 6-8.

Immunotherapy. Eight patients received a dosage of $1,000,000-3,000,000$ units of IFN $\alpha-2 b$ and 4 patients received $600,000-1,000,000$ units of IL-2. Continuous utilization of this dosage for 6-12 weeks equates to one biological cycle.

CIK adoptive immunotherapy. Peripheral blood (70 $\mathrm{ml})$ was collected from 5 patients 1 month after regular treatment. Mononuclear cells were isolated using the blood cell separation channel. Serum-free medium (AIMV) was used to adjust the cell count to $1 \times 10^{6} / \mathrm{ml}$. RhIFN-r $(1,000 \mathrm{U} / \mathrm{ml})$ was added to the medium on the first day. After $24 \mathrm{~h}$ of culturing, rhIFN-2 (300 U/ml), rhIFN-1 (300 U/ml) and OKT (350 ng/ml) were added and the medium was changed every 4 days. The cell count was adjusted to $1 \times 10^{6} / \mathrm{ml}$ and $\mathrm{rhIFN}-2$ was adjusted to $300 \mathrm{U} / \mathrm{ml}$ during medium changes. Every 8 days, OKT was adjusted to $350 \mathrm{ng} / \mathrm{ml}$ when rhIFN-2 was adjusted. After 2 weeks of culturing without contamination with bacteria and fungus, CIK cells were collected and washed with normal saline three times. Human serum albumin (10\%) was added and normal saline was used to make $100 \mathrm{ml}$ cell suspension. The cell suspension was intravenously injected into the patients multiple times over 3 days. More than 2-6x10 ${ }^{6}$ cells were injected on each occasion. Ten minutes before the injection, an intramuscular (IM) injection of promethazine $(25 \mathrm{mg}$ ) or intravenous injection of dexamethasone $(5 \mathrm{mg})$ was administered.

\section{Results}

Clinical follow-up. Clinical follow-up of the 22 patients was performed for 1-10 years with an average of 4.4 years. After 2 years, 3 patients were lost to follow-up. Twelve patients succumbed to multiple metastases and malignant consumption. One patient succumbed due to cerebral hemorrhage 2 years after surgery. During the process of clinical follow-up, 3 patients in the treatment group ( 2 at stage IV and 1 at stage III) succumbed, yielding a survival rate of $86.4 \%(19 / 22)$. The 3-year survival rate was $42.1 \%(12 / 19)$ and the 5-year survival rate was $31.2 \%$ (6/19). Recurrence or metastasis of tumors did not occur for 3 years in the 5 patients receiving CIK adoptive immunotherapy.

Local recurrence, distant metastasis and prognosis of the tumor. Two patients had postoperative local recurrence of the tumor and surgical removal for the extended areas was 
performed for these cases. Multiple metastases occurred in 13 patients. In most cases, the metastatic sites were liver, lung and bone. Of the 13 patients, 12 patients with multiple metastases succumbed. Patients with a tumor size of $<2 \mathrm{~cm}$ had a better prognosis than those with a tumor size of $\geq 2 \mathrm{~cm}$ $(p=0.009)$ (Table I). Patients with a deeper infiltration of the tumor had poor prognosis. Prognosis was significantly different among the patients with $<1.5 \mathrm{~cm}$ infiltration, patients with $1.5-4.0 \mathrm{~cm}$ infiltration and patients with $>4.0 \mathrm{~mm}$ infiltration $(\mathrm{p}=0.000)$. Patients with more advanced tumors had a poor prognosis. Prognosis was significantly different among stage I-IV patients $(\mathrm{p}=0.000)$. The prognosis of patients with axillary lymph node metastasis was worse than that of patients without axillary lymph node metastasis $(\mathrm{p}=0.003)$.

Adverse reactions. A total of 19 patients developed bone marrow suppression (I-III degree) following chemotherapy. After treatment to increase the white blood cells, normal function of the bone marrow was resumed. Five patients treated with interferon or IL-2 experienced symptoms, such as chills and fever. After symptomatic treatment, the patients' condition returned to normal. Two patients treated with transfusion of CIK cells experienced slight chills and fever. After intravenous injection of dexamethasone $(5 \mathrm{mg}$ ) and treatment for defervescence, the patients' condition returned to normal.

\section{Discussion}

Melanoma of the finger is a highly malignant tumor with rapid disease progression. Acral malignant melanoma has a low incidence, accounting for $5 \%$ of total malignant melanomas (7). Pathological symptoms were characterized by acral splash melanoma, early radioactive growth, shallow invasion and less likelihood of metastasis. Early treatment normally results in a favorable prognosis. In the late stage, the tumor exhibited vertical growth, a high rate of lymph node metastasis and poor prognosis. Early clinical symptoms of these patients are mild and easily overlooked. The tumor is normally at a late stage when the patient seeks medical attention (8). Local lymphadenectasis was observed in the 22 patients included in this study and $59.1 \%(13 / 22)$ of the patients were confirmed to have metastasis after surgery. The results suggest that tumors in the majority of these patients were already in advanced stages when the diagnosis was confirmed, which is consistent with previous reports (9). In order to make an early diagnosis, provide early treatment and improve the survival period, a biopsy should be performed for all cases of suspected malignant melanoma of the finger that present with expanded areas of black patches, darkening of the patches and appearance of uneven borders. Excision biopsy is recommended, whereas electric coagulation and curettage are not recommended due to adverse stimulation.

Surgical treatment is crucial for malignant melanoma. The type of surgery used for malignant melanoma is dependent on the local size of the tumor, the degree of tumor infiltration and the results of auxiliary examination, e.g., ultrasonography or CT. For stage I or II patients, amputation should be performed and the principle of the surgery is the removal of extended areas surrounding the local tumor. For stage III patients, both amputation and axillary lymph node removal should be performed. For patients with lymphadenectasis accompanied by skin infiltration, local lymph nodes and subcutaneous tissues should be removed in a spindle manner. In the present study, swollen lymph nodes and surrounding subcutaneous tissue was completely removed for 13 patients with axillary lymphadenectasis. Small satellite lesions were randomly distributed around the swelling lymph nodes in 2 patients. In this case, subcutaneous tissues were removed from the areas within more than $3 \mathrm{~cm}$ of the lesions and the intermediate dermatome was used to cover the wound areas. For stage IV patients, a combination of amputation, axillary lymph node dissection and liver intervention should be adopted. Through axillary lymph node dissection and intervention of liver metastasis, the tumor load can be reduced in advanced stage patients, long-distance metastasis can be delayed and the survival rate as well as the prognosis can be improved. The type of surgery performed should be selected depending on the disease condition of each individual patient.

In most patients, malignant melanoma of the finger is already at an advanced stage when diagnosed. Biopsy examination indicates that metastasis in malignant melanoma of the finger occurs early. Thus, therapies other than comprehensive surgical treatment are an important means of extending the life of patients with advanced stages of the disease. For example, chemotherapy drugs, such as CCNU, DTIC and DDP, achieved a relatively good efficacy. Utilization of the chemotherapy regimen with DTIC and DDP as the key drugs increases the therapeutic efficacy for malignant melanoma. In this study, one cycle of pre-operative chemotherapy and 4-6 cycles of postoperative chemotherapy (BDVP scheme) were found to reduce metastasis and recurrence after surgery and improve therapeutic efficacy.

Immunotherapy becomes increasingly attractive in the treatment of malignant tumors. Since the previous century, immunotherapy has been administered for malignant melanoma. Numerous studies have shown $(10,11)$ that application of BCG or IL-2 for autologous tumors reduces the recurrence and increases the survival rate without obvious intolerable side effects. Some of the patients in this study received IFN $\alpha-2 b$ and IL-2 treatment, and the findings showed that tumor recurrence and metastasis were effectively controlled. However, certain side effects occurred, such as fever, damage to liver and kidney function, and decrease of hemogram. Cytokineinduced killer (CIK) cell therapy is a new generation of adoptive $\mathrm{T}$ cell immunotherapy. The CIK cell is the main effector cell attacking tumor cells. Adoptive immunotherapy, including LAK and TIL, is achieved by the amplification and activation of antitumor $\mathrm{T}$ lymphocytes in vitro and transfusion of these activated cells back to the patients to kill the tumor cells. CIK cells are obtained by the utilization of cytokines and the optimization of culture conditions. CIK cells are tumor-specific $\mathrm{T}$ lymphocytes and are capable of effectively attacking tumors. At present, CIK cell therapy achieves $60-90 \%$ cytotoxic activity in the treatment of kidney cancer, malignant melanoma, leukemia and malignant lymphoma. Adoptive immunotherapy has become one of the supplemental approaches for tumor treatment. It has provided a novel therapeutic means to prevent tumor recurrence, eliminate residual tumor cells and improve quality of life for patients with an advanced stage tumor (12). Five patients in this study received CIK adoptive immunotherapy after chemotherapy. In the 
3-year follow-up, no recurrence or metastasis of the tumor occurred. These results indicate that adoptive immunotherapy with CIK cells after surgery and chemotherapy plays a crucial role in eliminating residual tumor cells and reducing tumor recurrence in patients with malignant melanoma.

\section{References}

1. Wen T, Junhui Z and Kefei Y: Diagnosis of subungual melanoma. Chin J of Hand Surg 9: 159-161, 1993.

2. Gogas HJ, Kirkwood JM and Sondak VK: Chemotherapy for metastatic melanoma: time for a change. Cancer 109: 455-464, 2007.

3. Atkins MB: Cytokine-based therapy and biochemotherapy for advanced melanoma. Clin Cancer Res 12: 2353-2358, 2006.

4. Khayat D, Bernard-Marty C, Meric JB and Rixe O: Biochemotherapy for advanced melanoma: maybe it is real. J Clin Oncol 20: 2411-2414, 2002.

5. Yongfu SH, Chaohong Y, Fusheng L, et al: Subungual melanoma: 30 case report. Chin J Oncol 11: 380-381, 1989.
6. Tseng JF, Tanabe KK, Gadd MA, et al: Surgical management of primary cutanomas of the hands and feet. Ann Surg 225: 544-553, 1997.

7. Morton DL: Immune response to postsurgical adjuvant active immunotherapy with Canvaxin polyvalent cancer vaccine: correlations with clinical course of patients with metastatic melanoma. Dev Biol 116: 209-217, 2004.

8. Baosheng Q and Qingtao Y: Diagnosis and treatment of subungual melanoma of finger. Chin J Hand Surg 17: 190, 2001.

9. Durán García E, Santolaya R and Requena T: Treatment of malignant melanoma. Ann Pharmacother 33: 730-738, 1999.

10. Wang Y, Cen Y and Li Z: Therapeutic result of operation combined with large-dose of roferon-a for cutaneous malignant melanoma. Chin J Reparative Reconstructive Surg 21: 37-39, 2007.

11. Hersey P: Active immunotherapy with viral lystates of micrometastasis following surgical removal of high melanoma. World Surg 16: 251, 1992.

12. Hofmen DM, Gitlita Hl, Belldegrun A and Figlin RA: Adoptive cellular therapy. Semin Oncol 27: 221-233, 2000. 